\title{
Prognostic factors for ESD of early gastric cancers: a systematic review and meta-analysis
}

\section{다 (ㄱ) $\odot$}

Authors

Michele Oliveira De Marco ${ }^{1}$, Francisco Tustumi ${ }^{2}$, Vitor Ottoboni Brunaldi ${ }^{3}$, Ricardo Hannum Resende ${ }^{1}$, Carolina Ogawa Matsubayashi ${ }^{1}$, Elisa Ryoka Baba ${ }^{1}$, Dalton Marques Chaves ${ }^{1}$, Wanderley Marques Bernardo ${ }^{4}$, Eduardo Guimarães Hourneaux de Moura' ${ }^{1}$

Institutions

1 Gastrointestinal Endoscopy Unit, Gastroenterology Department, University of São Paulo Medical School, São Paulo, Brazil

2 Department of Gastroenterology, Digestive Surgery Division, Sao Paulo School of Medicine

3 Gastrointestinal Endoscopy Unit, Gastroenterology Department, University of São Paulo Medical School, São Paulo, Brazil; Center for Gastrointestinal Endoscopy, Surgery and Anatomy Department, Ribeirão Preto Medical School, University of São Paulo, Ribeirão Preto, Brazil

4 University São Paulo Medical School - São Paulo, Brazil Thoracic Surgery Department

submitted 18.9.2019

accepted after revision 12.2 .2020

Bibliography

DOI https://doi.org/10.1055/a-1201-3089 |

Endoscopy International Open 2020; 08: E1144-E1155

(c) Georg Thieme Verlag KG Stuttgart · New York

eISSN 2196-9736

Corresponding author

Michele Oliveira De Marco, Department of

Gastroenterology, Faculty of Medicine, University of

São Paulo, Dr Eneas Carvalho De Aguiar Avenue 255,

05403-000 São Paulo - SP, Brazil

michelemarco@gmail.com

Supplementary material

Online content viewable at:

https://doi.org/10.1055/a-1201-3089

\section{ABSTRACT}

Background and study aims Gastric neoplasms are one of the leading types of cancer in the world and early detection is essential to improve prognosis. Endoscopy is the gold-standard diagnostic procedure and allows adequate treatment in selected cases. Endoscopic submucosal dissection (ESD) has been reported to safely address most ear- ly gastric cancers (EGCs), with high curability rates. However, data on prognostic factors related to ESDs of EGCs are conflicting. Therefore, we aimed to systematically review the available literature and to perform a meta-analysis to identify the relevant prognostic factors in this context. Methods We performed this study according to PRISMA guidelines. Comparative studies assessing the relationship between curative resection or long-term curability rates and relevant prognostic factors were selected. Prognostic factors were demographic data, lesion features (location, morphology of the lesion, size, and depth of invasion), histological findings, Helycobacter pylori (HP) infection, presence of gastric a atrophy and body mass index (BMI). Finally, we also evaluated risk factors related to metachronous gastric neoplasm.

Results The initial search retrieved 2829 records among which 46 studies were included for systematic review and meta-analysis. The total sample comprised 28366 patients and 29282 lesions. Regarding curative resection, pooled data showed no significant influence of sex [odds ratio (OR): $1.15(0,97,1.36) P=0.10 \mathrm{I}^{2}=47 \%$ ] , age [OR: 1.00 $\left.(0.61,1.64) P=1.00 I^{2}=58 \%\right]$, posterior vs non-posterior location [OR: $1.35(0.81,2.27) P=\left.0.25\right|^{2}=84 \%$ ], depressed vs von-depressed macroscopic type[OR: $1.21(0.99,1.49) P=$ $0.07 \mathrm{I}^{2}=0 \%$, non-upper vs upper location [OR: $1.41(0.93$, 2.14) $P=0.10 I^{2}=77 \%$ ] and BMI [OR: $0.84(0.57 ; 1.26) P=$ $0.41 \mathrm{I}^{2}=0 \%$. Differentiated neoplasms presented greater chance of cure compare to undifferentiated [OR: 0.10 $\left.(0.07,0.15) \quad P<0.00001 I^{2}=0 \%\right]$. Ulcerated lesions had lower curative rates compared to non-ulcerated [OR: 3.92 $\left.(2.81,5.47) P<0.00001 \mathrm{I}^{2}=44 \%\right]$. Lesions smaller than $20 \mathrm{~mm}$ had greater chance of curative resection [OR: 3.94 $(3.25,4.78) P<0.00001 \mathrm{I}^{2}=38 \%$. Bleeding during procedure had lower curative rates compared to non-bleeding [OR: $2.13(1.56,2.93) P<0.0001 \mathrm{I}^{2}=0 \%$ ]. Concerning longterm cure, female gender [OR $1.62(1.33,1.97) P<0.00001$ $\left.\mathrm{I}^{2}=0 \%\right]$ and the mucosal over SM 1 cancers were protective factors [OR: $0.08(0.02,0.39) P=0.002 \mathrm{I}^{2}=86 \%$ ]. Gastric atrophy [OR: $0.60(0.45,0.81) P=0.0006 I^{2}=42 \%$ ] and the pepsinogen I/pepsinogen II ratio [OR $2.29(1.47,3.57) P=$ $0.0002 \mathrm{I}^{2}=0 \%$ ] were risk factors to metachronous gastric neoplasm. 
Conclusions Ulcerated lesions, histology, bleeding and size $>20 \mathrm{~mm}$ are prognostic factors concerning curative resection. Regarding long-term cure, female gender and mu- cosal over SM1 cancer are predictive factors. Gastric atrophy and the pepsinogen ratio are risk factors for metachronous gastric neoplasm.

\section{Introduction}

Gastric cancer is a major worldwide health problem. It is the fourth most common cancer and the second leading cause of cancer death worldwide. More than 950,000 new diagnoses occur annually. It is estimated that 720,000 patients died of gastric cancer in 2012 [1].

Early diagnosis of gastric cancer is of economic significance because expenses related to advanced cancer treatment are usually high. Cancer treatment accounts for $4 \%$ to $10 \%$ of total health costs worldwide, with the ever-increasing costs widely seen as unsustainable from a public health perspective [2].

Upper endoscopy is the method by which detection is possible, allowing for adequate treatment. Endoscopic submucosal dissection (ESD) is considered the gold standard for cure of early gastric cancer [3-6]. Kondo et al. [7] established that in a meta-analysis that compared endoscopic and surgical treatment for early gastric cancer. Results revealed that in regards to survival, surgery and endoscopy have similar outcomes. However, endoscopy has lower risk of adverse events (AEs), as well as less morbidity, preserves gastric anatomy and promotes better long-term quality of life. Therefore, endoscopic treatment is considered the first choice for treatment of early gastric cancer [8-12]. A gastric tumor that does not extend beyond the submucosal layer is defined as early gastric cancer, regardless of presence or absence of lymph node metastasis $[6,7,13]$.

Limited data are available in the literature on evaluation of prognostic factors related to endoscopic treatment of early gastric cancer. Some researchers have postulated that the topography of certain tumors prevents resection and curability, as there is doubt regarding performance of such procedures in elderly patients or tumors of undifferentiated histology. Minimally invasive treatment is part of current practice. Knowledge of prognostic factors associated with it is as important as diagnostics for proper selection of therapy and individualized management.

We sought to identify the main prognostic factors related to ESD for treatment of early gastric cancer through a systematic review and meta-analysis of currently available literature. As a secondary objective, we aimed to evaluate factors associated with incidence of metachronous tumor during follow-up, after curative resection of the primary tumor.

\section{Methods}

\section{Database search}

A systematic and structured search was carried out through the PICO system up to June 2019 [14]. MEDLINE, Embase, Web of Science, OVID, Cochrane, Scopus, LILACS/Bireme, Ageline/ CINAHL/EBSCo and CAPES were used, as well as the grey litera- ture (hand-searching and scanning reference lists). Two independent reviewers using predefined inclusion and exclusion criteria performed an eligibility assessment and selection of studies identified in the primary search. If reviewers disagreed about inclusion or exclusion of a given study, eligibility was decided in a consensus meeting. Cohort observational studies and case series were included.

Search strategy in MEDLINE: (gastric OR stomach) AND (tumor OR tumors OR tumour OR tumours OR tumoral OR cancer OR cancers $O R$ cancerous OR neoplasm* OR adenoma*) OR (Neoplasm, Stomach OR Stomach Neoplasm OR Neoplasms, Stomach OR Gastric Neoplasms OR Gastric Neoplasm OR Neoplasm, Gastric OR Cancer of Stomach OR Stomach Cancers OR Gastric Cancer OR Cancers, Gastric OR Stomach Cancer OR Cancers, Stomach OR Cancer of the Stomach) AND ((endoscopic mucosal resections OR mucosal resection, endoscopic OR mucosal resections, endoscopic OR strip biopsy $O R$ biopsies, strip OR endoscopic mucous membrane reaction $O R$ endoscopic submucosal dissection $O R$ dissections, endoscopic submucosal OR endoscopic submucosal dissection $O R$ EMR OR ESD ).

Search strategy in EMBASE: 'stomach cancer' AND 'endoscopic submucosal dissection' AND [embase]/lim NOT [medline]/lim.

In the remaining databases, search strategies were derived from those aforementioned with simplifications due to intrinsic limitations of the search tools.

This study was recorded in The International Prospective Register of Systematic Reviews-University of York- PROSPERO: CRD42018115754) and approved by our institution's ethics committee.

\section{Inclusion criteria:}

1. Adults over 18 years

2. Patients who underwent ESD for resection of early gastric cancer

3. Studies that assessed prognostic factors associated with curability and clinical outcomes.

\section{Exclusion criteria:}

1. Reviews, editorials, letters and conference summaries

2. Animal studies

3. Studies without full text in English

4. Studies in which data could not be extracted

5. Studies that evaluated cure without reporting follow-up.

\section{Quality verification of studies:}

Study quality was evaluated according to NewCastle-Ottawa [15]. The items analyzed by this scale include: (1) Selection criteria: representativeness of the included cohort, selection of the unexposed cohort, the ascertainment of the exposure, demonstration that the expected result was not present at the 
beginning of the study; (2) Comparability criteria: comparability of the cohort based on the analysis. (3) Outcome criteria: Analysis of results and adequate follow-up.

\section{Statistical analysis:}

Absolute numbers were extracted and differences in risk or odds ratio from dichotomous variables for each outcome were analyzed using Review Manager Software Version 5.3 (Copenhagen, the Nordic Cochrane Center, The Cochrane Collaboration, 2014). Both fixed and random effects models were used depending on the findings of heterogeneity between studies. The Mantel-Haenszel test was used for categorical variables and inverse variance was used for continuous variables. Meta-analysis results were expressed in forest plot graphs. Funnel plot graphics were used to identify publication bias, as explained below.

\section{Additional analysis:}

The cut-off value of $30 \%$ heterogeneity was considered adequate for this meta-analysis. Thus, sensitivity analysis was performed when the heterogeneity measured by the Higgins test $\left(I^{2}\right)$ was higher than $30 \%$. A subsequent analysis was performed, which excluded possible outliers. When outliers were not detected, true heterogeneity was presumed with exclusion of publication bias and random analysis model was assumed.

\section{Definitions}

\section{Indications for ESD}

According to the Japanese Endoscopy Society [16], ESD is indicated as follows:

Absolute criterion: differentiated intramucosal carcinoma of less than $2 \mathrm{~cm}$, independent of macroscopy, preferably without ulcer (UL-).

Expanded criteria: (1) differentiated intramucosal carcinoma greater than $2 \mathrm{~cm}$; (2) differentiated carcinoma less than $3 \mathrm{~cm}$, which may be ulcerated (UL+); (3) Undifferentiated carcinoma of less than $2 \mathrm{~cm}$ and without ulcer (UL-).

\section{Curative resection}

Studies that matched the inclusion criteria defined curative resection as one in which indications for ESD were associated with absence of venous, neural or lymphatic invasion and free margins after histological evaluation.

Cure

Similar to the above, studies that met inclusion criteria defined cure as curative resection with no evidence of local or metastatic recurrence during follow up.

\section{Metachronous tumor}

Metachronous tumor was defined as a new early gastric tumor 1 year after treatment of the primary site, in a different location, and that was diagnosed during follow-up.
Histological, macroscopic, and topographic classification

The current classifications are in accordance with the third edition of the Japanese classification of gastric carcinoma [3]. For histological type, this classification subdivides tumors into more than 20 subtypes, listed in the supplementary material (Supplementary Table 1). In this study, we evaluated the histological types mentioned in the included studies, which are differentiated adenocarcinoma, undifferentiated adenocarcinoma, and undifferentiated tumor with signet ring cells. The Japanese classification divides early neoplasms, called superficial tumors (Type 0), into: (1) Polypoid (Type 0-I), (2) Flat-slightly elevated (Type 0-Ila), (3) Superficial flat (Type 0-IIb), (4) Superficial depressed (Type 0 -IIc), (5) Tumors with deep depression, excavated or ulcerated (Type 0-III). Concerning topography, this classification divides tumor location according to the longitudinal and transverse axis of the stomach, with the divisions of the longitudinal axis: upper, middle and lower third; and those of the transverse axis: anterior wall, posterior wall, small curvature and greater curvature. Schematic figure is found in the supplementary material (Supplementary Fig. 1).

\section{Definition of Obese}

Used as referred by the Consensus Statement for Diagnosis Of Obesity In Asian populations [17]: (1) normal: BMI $<23 \mathrm{~kg} / \mathrm{m}^{2}$, (2) overweight: $23 \mathrm{~kg} / \mathrm{m}^{2} \leq \mathrm{BMI}<25 \mathrm{~kg} / \mathrm{m}^{2}$, (3) obese: BMI $\geq 25 \mathrm{~kg} / \mathrm{m}^{2}$

\section{Results}

The initial search retrieved 4050 records, of which, after evaluation of titles and abstracts, 146 articles were selected. After reading the full text individually, 46 studies were selected to be included in the systematic review and meta-analysis. The total sample comprised 28366 patients and 29282 lesions ( Fig.1). All included studies achieved excellent quality (score $>6$ ).

The articles were divided into two groups: those that evaluated curative resection associated with prognostic factors and without follow-up; and those that included long-term follow-up, allowing for characterization of cure.

\section{Curative resection}

Data were extracted from 19 articles including 13704 patients and 14468 lesions ( $>$ Table $\mathbf{1}$ ), correlating curative resection with prognostic outcomes. Tables with the correlations of all prognostic outcomes are included (Supplementary Table 2, Supplementary Table 3).

Gender - There was no significant difference in relation to curative resection between genders [Odds Ratio (OR): 1.15 $\left.(0,97,1.36) P=0.10 I^{2}=47 \%\right]$ (Supplementary Fig. 2)

Age - There was no significant difference in relation to curative resection between elderly and non-elderly patients. Sensitivity analysis did not show any outlier, with heterogeneity being considered as true and considered a random effect [OR: $\left.1.00(0.61,1.64) P=1.00 \mathrm{I}^{2}=58 \%\right]$. Two articles in this review (Iwai $\mathrm{N}$ et al and Katsube $\mathrm{T}$ et al) considered elderly people as those over 80 years old and one (Kato $\mathrm{M}$ et al) considered over 


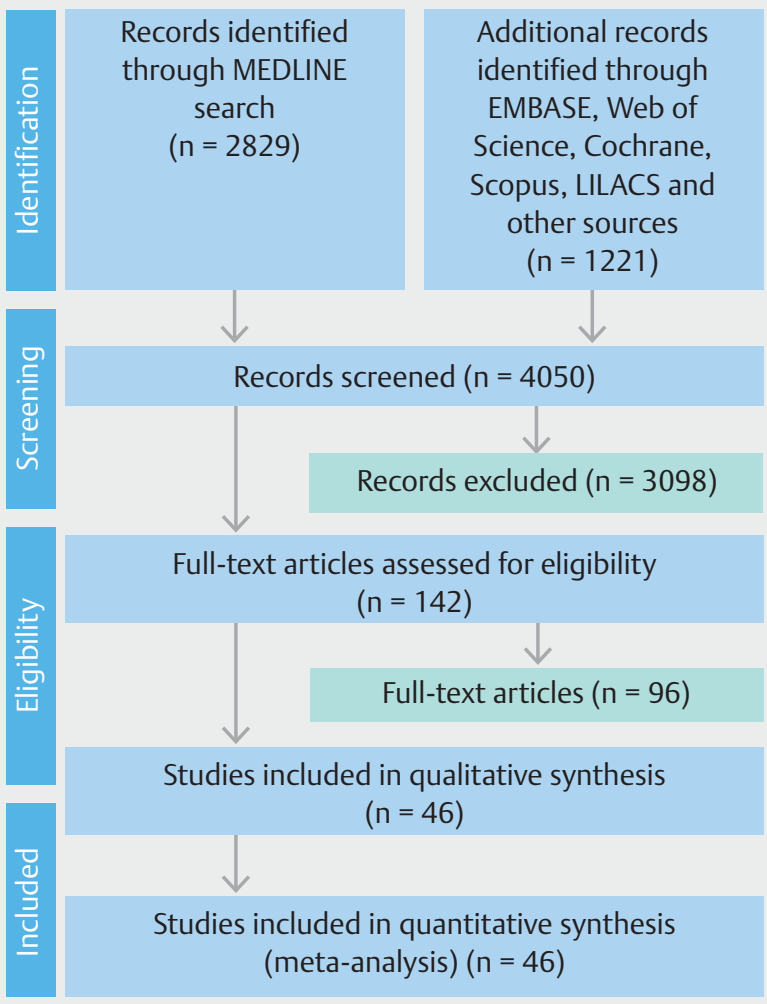

Fig. 1 PRISMA Chart.

75 years. This article, when excluded from the analysis, did not affect the results, which showed no statistical significance ( Fig. 2).

$\mathrm{BMI}$ - There was no difference in the curative resection when comparing obese and non-obese individuals [OR: 0.84 (0.57; 1.26) $P=0.41 \mathrm{I}^{2}=0 \%$ ] (Supplementary Fig. 3).

Helicobacter pylori infection-There was a significant statistical difference in the curative resection of patients with $\mathrm{H}$. pylori infection [OR: $0.63(0.49,0.80) P=0.0002 \mathrm{I}^{2}=48 \%$ ] (Supplementary Fig. 4).

Histological type - Differentiated neoplasms have a greater chance of curative resection in relation to undifferentiated ones, with statistical significance. The sensitivity assessment identified an outlier, which was eliminated for the final analysis [OR: $0.10(0.07,0.15) P<0.00001 \mathrm{I}^{2}=0 \%$ ] (Supplementary Fig. 5).

Location - In relation to the longitudinal axis, the analysis showed a statistical significance for curative resection of cancers located in the middle and distal third (not superior), however, with high heterogeneity [OR: $1.62(1.36,1.93) P<$ $\left.\left.0.00001 \mathrm{I}^{2}=77 \%\right)\right]$. The sensitivity analysis revealed an outlier, with a high heterogeneity even after its elimination [OR: 1.34 $\left.\left.(1.06,1.68) P=0.01 \mathrm{I}^{2}=76 \%\right)\right]$. Therefore, heterogeneity was considered as true and another analysis was performed with random effect. After equalization, despite favoring tumors not located in the upper third, statistical significance was lost [OR: $\left.1.41(0.93,2.14) P=0.10 I^{2}=77 \%\right]$. (Supplementary Fig. 6).
In relation to the transverse axis, there was no statistical difference between the curative resection of tumors located in the posterior and non-posterior walls [OR: $1.35(0.81,2.27) P=$ $\left.0.25 \mathrm{I}^{2}=84 \%\right]$. The initial analysis revealed high heterogeneity, however, there was no outlier, and the random effect was used in the final analysis. (Supplementary Fig. 7).

Macroscopic type - There was no statistical difference between depressed and non-depressed macroscopic types [OR: $1.21(0.99,1.49) P=0.07 I^{2}=0 \%$ ] (Supplementary Fig. 8).

Ulcerative lesions - Non-ulcerated tumors have a greater chance of curative resection compared to ulcerated tumors, with statistical significance [OR: $3.92(2.81,5.47) P<0.00001$ $\left.\mathrm{I}^{2}=44 \%\right]$ (Supplementary Fig. 9).

Size - Lesions smaller than $20 \mathrm{~mm}$ had a greater chance of curative resection [OR: $4.16(3.44,5.03) P<0.00001 \mathrm{I}^{2}=67 \%$ ]. The sensitivity assessment identified an outlier, which was eliminated for the final analysis [OR: $3.94(3.25,4.78) P<0.00001$ $\mathrm{I}^{2}=38 \%$ ] (Supplementary Fig. 10).

Depth - Lesions limited to the submucosa or superficial mucosa (SM1) had a greater chance of curative resection. Sensitivity analysis did not identify any outlier [OR: $0.02(0.00,0.69) P=$ $0.03 \mathrm{I}^{2}=95 \%$ ] (Supplementary Fig. 11).

Bleeding - Gastric cancers that do not present with bleeding during endoscopic resection have a greater chance of curative resection [OR: $2.13(1.56,2.93) P<0.0001 I^{2}=0 \%$ ] ( Fig. 3).

\section{Long-term results}

Data were extracted from 27 articles ( $\triangleright$ Table 2 ) with analysis of 14,662 patients and 14,814 lesions. Cure was correlated with prognostic outcomes presented in each study. Tables with all prognostic factors are found in the supplementary material. We also extracted data on incidence of metachronous tumor during follow-up, correlating its presence with Helicobacter pylori infection, degree of gastric atrophy and pepsinogen ratio I and II. Mean follow-up time was 44.85 months (Supplementary Table 5, Supplementary Table 6)

Gender - Women had a greater chance of long-term cure compared to men [OR $\left.1.62(1.33,1.97) P<0.00001 \mathrm{I}^{2}=0 \%\right]$ (Supplementary Fig. 12).

Age - Regarding long-term cure, there was no difference between the elderly and the non-elderly. One of the articles (NAM HS et al) defined the elderly as being older than 65 years. Others defined elderly as being over 75 years old. Analysis of sensitivity did not affect heterogeneity, which is why the random effect was considered for final analysis [OR: $1.49(0.69,3.23) P=$ $0.31 \mathrm{I}^{2}=63 \%$ ] (Supplementary Fig. 13).

Histology - There was no difference in long-term cure of differentiated and undifferentiated tumors [OR: $0.71(0.20,2.51)$ $P=0.60 \mathrm{I}^{2}=89 \%$. Sensitivity analysis was performed, with high heterogeneity remaining after elimination of the outlier, and, therefore, the random effect was used in the analysis (Supplementary Fig. 14).

There was also no difference in long-term cure between undifferentiated tumors: undifferentiated adenocarcinoma (PDA) and carcinoma with signet ring cells (SRC). Due to the high heterogeneity, a random effect was used for OR analysis: [OR: 2.24 $\left.(0.44,11.35) P=0.33 \mathrm{I}^{2}=87 \%\right]$ (Supplementary Fig. 15). 
- Table 1 Curative resection with prognostic outcomes.

\begin{tabular}{|c|c|c|c|c|c|}
\hline & Country & Patients & Lesions & Male & Female \\
\hline Horiuchi Y 2017 & Japan & 268 & 268 & $25 / 152$ & $14 / 116$ \\
\hline Horiuchi Y 2018 & Japan & 2551 & 2585 & $288 / 2008$ & $66 / 577$ \\
\hline Choi JM 2016 & South Korea & 1615 & 1641 & & \\
\hline Iwai N 2018 & Japan & 585 & 708 & & \\
\hline Horiuchi Y, Fujisaki J 2018 & Japan & 81 & 81 & & \\
\hline Yoon JY 2014 & South Korea & 1319 & 1443 & & \\
\hline Numata N 2013 & Japan & 63 & 79 & & \\
\hline Kim EH 2017 & Korea & 1639 & 1670 & $193 / 1211$ & $79 / 428$ \\
\hline Libânio D 2017 & Portugal & 164 & 194 & $21 / 104$ & 009/90 \\
\hline Kato M 2016 & Japan & 892 & 1062 & & \\
\hline Choill 2016 & Korea & 712 & 737 & $77 / 584$ & $20 / 173$ \\
\hline Katsube T 2015 & Japan & 231 & 231 & $40 / 178$ & $14 / 53$ \\
\hline Toyokawa T 2015 & Japan & 967 & 1123 & $49 / 723$ & $010 / 277$ \\
\hline Sanomura Y 2014 & Japan & 78 & 94 & & \\
\hline Shindo Y 2016 & Japan & 250 & 262 & & \\
\hline Choi YK 2018 & Korea & 316 & 316 & & \\
\hline Nakanishi H 2016 & Japan & 760 & 760 & & \\
\hline Tanaka S 2014 & Japan & 32 & 33 & & \\
\hline Kang D 2017 & Korea & 1181 & 1181 & & \\
\hline
\end{tabular}

Macroscopic type - There was also no significant difference when comparing depressed and non-depressed macroscopic types [OR: $1.12(0.72,1.74) P=0.60 I^{2}=66 \%$ ], as the presence of ulcer was not statistically significant associated with cure [OR: $0.91(0.21,3.95) P=0.09 \mathrm{I}^{2}=0 \%$ ] (Supplementary Fig. 16).

Location - Although favoring inferior localization, location was not significant when comparing tumors from upper and lower two thirds [OR: $1.25(0.97,1.63) P=0.09 \mathrm{I}^{2}=0 \%$ ) (Supplementary Fig. 17).

Size - Tumors larger or smaller than $20 \mathrm{~mm}$ [OR: 1.20 (0.93, 1.55) $\left.P=0.16 \mathrm{I}^{2}=74 \%\right)$ or $30 \mathrm{~mm}$ [OR: $1.63(0.89,2.97) P=0.11$ $\left.\mathrm{I}^{2}=77 \%\right]$ did not show any statistical significance regarding long-term cure (Supplementary Fig. 18)

Depth - Tumors that invade less than 500 microns of submucosa (SM1) have a greater chance cure rate than those that go more deeply [OR: $0.08(0.02,0.39) P=0.002 \mathrm{I}^{2}=86 \%$ ]. The sensitivity analysis did not change significantly the heterogeneity, so the random effect was used in the final analysis. (Supplementary Fig. 19).

\section{Metachronous tumor}

Gender - The analysis showed that the female gender is a protective factor [OR: $1.64(1.32,2.03) P<0.00001 \mathrm{I}^{2}=0 \%$ ] (Supplementary Fig. 20).
H. pylori-infection status was not relevant per se at onset of metachronous tumor. We analyzed patients with eradicated and persistent $H$. pylori [OR: $1.37(0.95,1.97) P=0.09 \mathrm{I}^{2}=0 \%$ ] and found no statistical significant difference between them (Supplementary Fig. 21).

The analysis of persistent and negative $H$. pylori infection had high heterogeneity, with no evidence of any outlier. We assumed the heterogeneity to be true and used the random effect in the analysis, which did not have statistical difference [OR: $1.61(0.90,2.89) P=0.11 \mathrm{I}^{2}=69 \%$ ] (Supplementary Fig. 22).

There was also no significance between incidence of metachronous tumor between the group that was always $H$. pylori negative and the group that became negative after eradication. Sensitivity analysis did not allow for removal of outliers, so a random effect was used. [OR $0.88(0.21,3.61) P=0.85 \mathrm{I}^{2}=$ $82 \%]$ (Supplementary Fig. 23).

Gastric atrophy - Degree of atrophy was related to the incidence of metachronous tumor, with a lower risk of progression being associated with less severe atrophy. [OR: $0.60(0.45$, 0.81) $P=0.0006 \mathrm{I}^{2}=42 \%$ ] ( Fig. 4).

Pepsinogen - The ratio between pepsinogen I and II greater than 3 is a protective factor when considering incidence of a new cancer [OR $2.29(1.47,3.57) P=0.0002 \mathrm{I}^{2}=0 \%$ ) (Supplementary Fig. 24), > Table 3 summa the results of this study. 


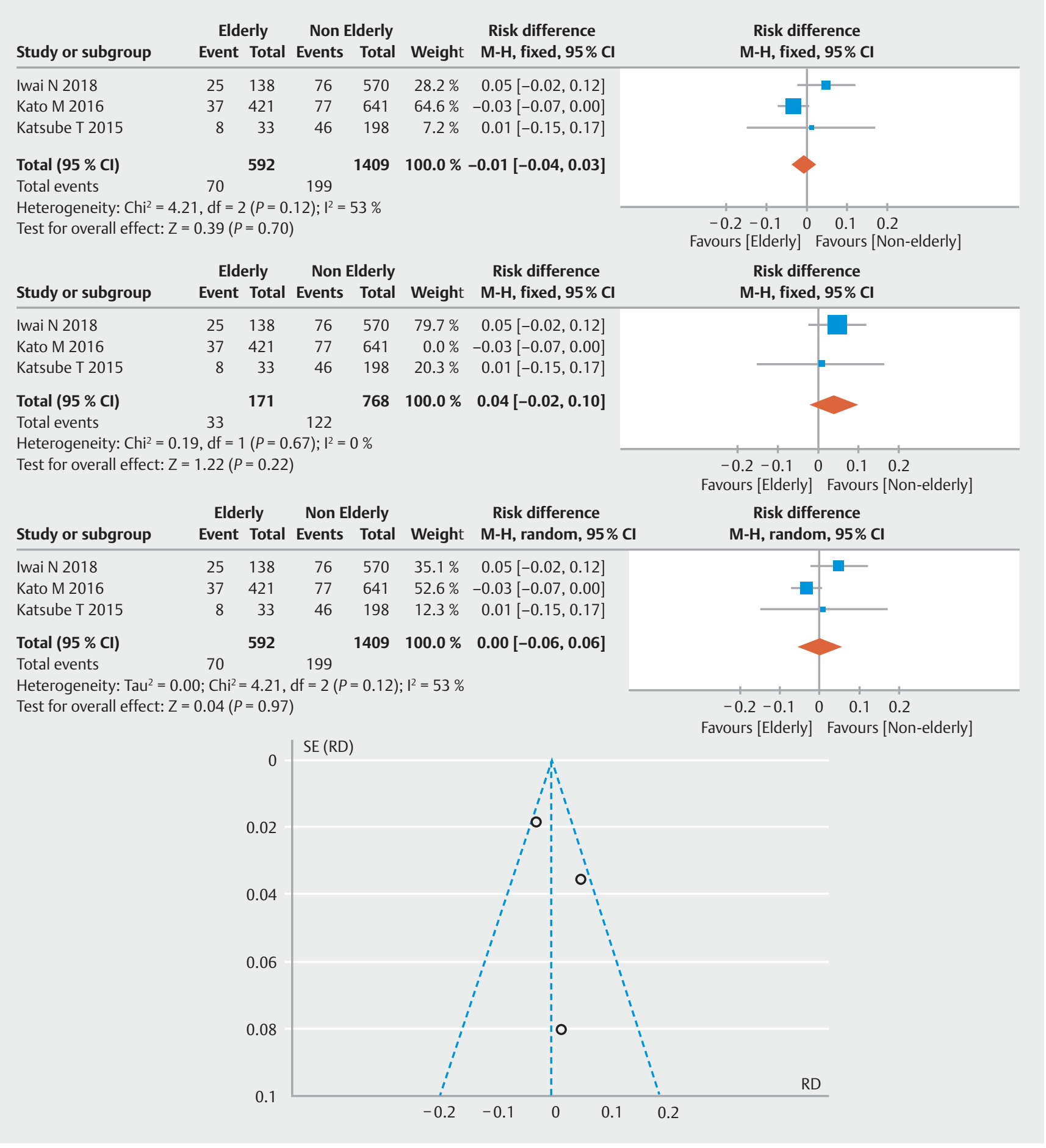

- Fig. 2 Graphs analyzing age in curative resection. There is no significant difference.

\section{Discussion}

This is the first systematic review in the literature that simultaneously evaluates several groups of prognostic factors in endoscopic treatment of early gastric cancer. This effort has great clinical relevance because identification of unfavorable factors allows for a more precise discussion of the indication for endo- scopic resection and also allows for individualization of followup based on real risk of relapse.

Male gender and advanced age are risk factors for gastric cancer [1]. There was no statistical difference between genders when the analysis considered only curative resection, but female gender was considered a protective variable for longterm results and for risk for metachronous tumor. Also, there 


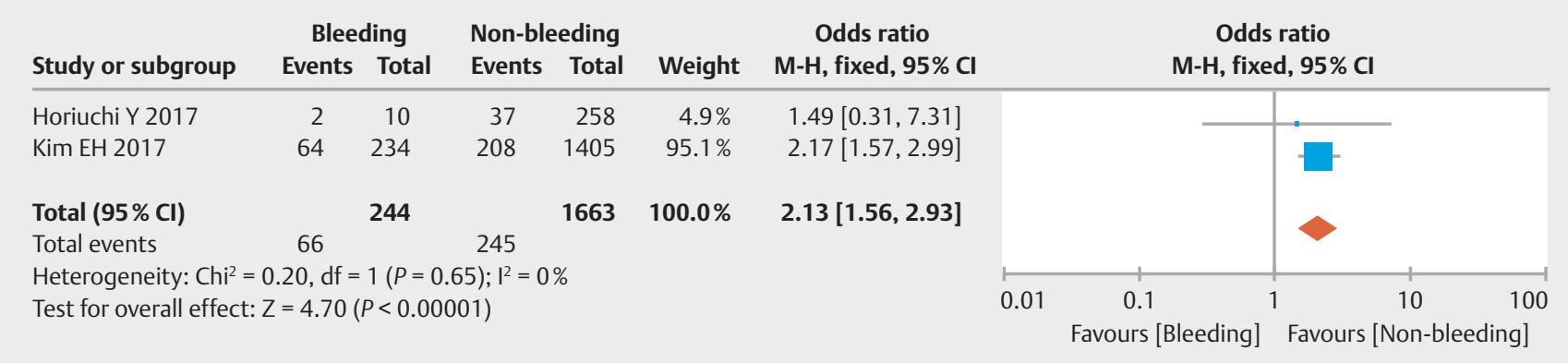

Fig. 3 Forest plot analyzing bleeding in curative resection.

- Table2 Cure: Prognostic outcomes presented in each study.

\begin{tabular}{|c|c|c|c|c|c|c|}
\hline & Country & N Patients & N Lesion & Follow-up median (mo) & Male & Female \\
\hline Jeon HK 2018 & Korea & 66 & 66 & 40 & & \\
\hline Bang CS 2017 & Korea & 275 & 275 & 47 & & \\
\hline Zhang Y 2014 & China & 171 & 187 & 27.5 & & \\
\hline Sumiyoshi T 2017 & Japan & 177 & 209 & 79 & $27 / 118$ & $005 / 59$ \\
\hline NAM HS 2018 & Korea & 639 & 639 & 36.2 & $13 / 502$ & $002 / 137$ \\
\hline Isomoto H 2010 & Japan & 661 & 713 & 30 & & \\
\hline Goto A 2017 & Japan & 423 & 423 & 61 & $007 / 343$ & $002 / 80$ \\
\hline Toyokawa T 2011 & Japan & 514 & 586 & 26.7 & & \\
\hline Lee JY 2016 & Korea & 401 & 415 & 19.7 & $25 / 291$ & $11 / 124$ \\
\hline Goh PG 2011 & Korea & 210 & 210 & 19.3 & & \\
\hline Han SJ 2018 & Korea & 565 & 565 & 60 & $46 / 440$ & $004 / 125$ \\
\hline Yang HJ 2018 & Korea & 1115 & 1115 & 50 & & \\
\hline Yang HJ, Kim SG 2018 & Korea & 1237 & 1237 & 50.2 & & \\
\hline Kwon Y 2017 & Korea & 590 & 590 & 54.4 & $47 / 398$ & $017 / 192$ \\
\hline Kim SB 2016 & Korea & 433 & 433 & 35.6 & $015 / 325$ & $000 / 108$ \\
\hline Machata Y 2012 & Japan & 268 & 268 & 62.4 & $022 / 194$ & $006 / 74$ \\
\hline Iguchi M 2016 & Japan & 330 & 330 & 50.4 & $039 / 240$ & $008 / 90$ \\
\hline Park CH 2016 & Korea & 1447 & 1478 & 22.5 & & \\
\hline Moribata K 2015 & Japan & 122 & 122 & 46.8 & 017/091 & $005 / 31$ \\
\hline Abe S 2015 & Japan & 1526 & 1526 & 82.2 & $201 / 1180$ & $037 / 346$ \\
\hline Jung DH 2015 & Korea & 136 & 136 & 30.1 & $026 / 104$ & $008 / 32$ \\
\hline Min BH 2015 & Korea & 1306 & 1306 & 61 & $40 / 1044$ & $007 / 302$ \\
\hline Sugimoto T 2015 & Japan & 155 & 155 & 50.7 & $20 / 119$ & $003 / 36$ \\
\hline Chung CS 2014 & Korea & 283 & 183 & 44 & $23 / 190$ & $008 / 93$ \\
\hline Jung S 2015 & Korea & 1041 & 1041 & 42.6 & $23 / 773$ & $009 / 268$ \\
\hline Han JS 2011 & Korea & 176 & 176 & 34.6 & & \\
\hline Han JP 2014 & Korea & 395 & 430 & 47.3 & & \\
\hline
\end{tabular}




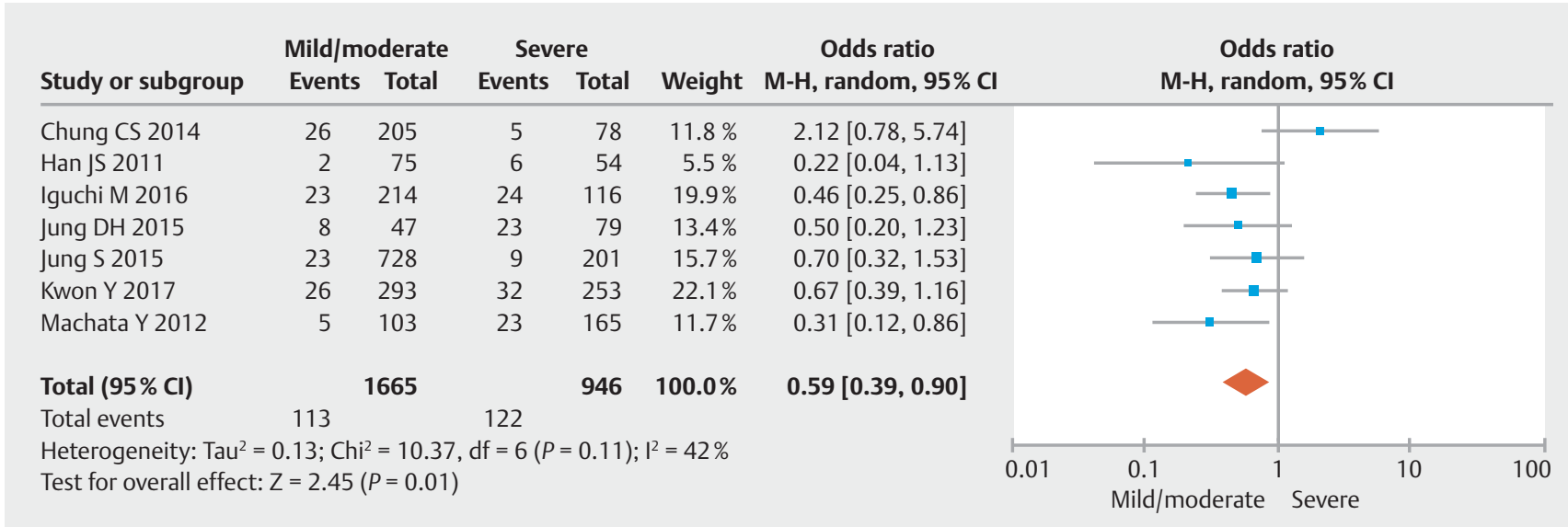

- Fig. 4 Graphs analyzing gastric atrophy in the incidence of metachronous tumors.

is evidence that gender may influence grade of cellular differentiation in gastric neoplasms [18].

There was no difference in relation to curative resection and cure between the elderly and the non-elderly. The precise definition of the elderly depends on the life expectancy of each geographic region. According to the Japanese Census of 2015 [19], life expectancy in Japan was 80 years for men and 86 years for women. This definition is the reason why two articles [20, 21 ] considered the elderly to be 80 years old. Five articles that we analyzed [22-26] used over 75 years as a definition of the elderly and one used over 65 years old [27]. There was no impact on results when we analyzed articles that evaluated subjects within the same age range, or even after exclusion of an article that defined elderly as 65 years old and above. Therefore, included all articles in the final analysis. Lin et al. published a meta-analysis that demonstrated efficacy and safety of gastric ESD in elderly patients, despite the high chance of pneumonia as a complication after the procedure [28]. Despite this frequent complication, technological advances in minimally invasive medical therapies such as ESD have contributed to increased life expectancy in the elderly population worldwide $[23,29]$. Interestingly, patient age does not impact prognosis of cancer. Thus, this factor should not be a criterion used routinely to contraindicate ESD in an elderly patient.

In regards to histological type, we were able to compare curative resection and cure of differentiated and undifferentiated tumors. Differentiation is a good prognostic factor when evaluating curative resection rate, but there was no statistical difference when evaluating long-term cure rate. High heterogeneity among studies, proven to be true after outlier removal, may have contributed to the absence of difference in long-term cure.

In 2000, Gotoda et al. [30-33] suggested the expanded ESD criteria after demonstrating that risk of lymph node metastasis was very low. This allowed for resection of undifferentiated non-ulcerated tumors smaller than $2 \mathrm{~cm}$. In the current study, we analyzed two articles that evaluated cure between two types of undifferentiated tumors: undifferentiated adenocarcinoma and signet ring neoplasia. There was no difference be- tween these two tumor types in relation to cure in our analysis. In the retrospective study by Jeon et al. [34] there was also no recurrence or metastasis in tumors resected, according to the expanded criteria. However, submucosa extension and size larger than $2 \mathrm{~cm}$ were the main predictors of incomplete resection, with similar results to the meta-analysis performed by Zhao et al. [35].

Some studies [36-38] evaluated undifferentiated early gastric cancer that had a mixed component (signet ring associated with areas of undifferentiated adenocarcinoma or foci of undifferentiated neoplasm in differentiated tumors) and concluded that these mixed neoplasms have a higher risk of non-curative endoscopic treatment, independent of other factors. However, absence of studies that met our inclusion criteria did not allow for this meta-analysis to evaluate this prognostic factor (mixed tumors).

A meta-analysis performed by Bang et al. [39] evaluated overall safety of ESD for early gastric cancer with undifferentiated histology, based on the expanded criteria, according to AEs that occurred during the procedure (gastric hemorrhage and perforation). Rates of gastric hemorrhage and perforation from the procedure were estimated to be $6.7 \%$ (95\% Cl: $4.1 \%-$ $10.8 \%, P<0.1)$ and $4.8 \%(95 \% \mathrm{Cl}: 2.6 \%-8.6 \%, P<0.1)$, respectively. However, our study evaluated bleeding potential as a predictor of poor prognosis in gastric ESD. We found that bleeding decreases rate of curative resection. Such results are possibly related to hindered visualization that may prevent more precise assessment of lesion boundaries, increasing chance of residual lesion or compromised margins.

Tumor location is one of the most important factors associated with absence of complications and resection success [40-42]. However, few studies and no meta-analyses have evaluated ESD curability outcomes according to location in the longitudinal and transverse planes, as mentioned in the Japanese classification of gastric cancer [3]. Regarding curative resection, it is more likely to be successful in tumors located in the middle and distal portions of the stomach. Regarding the division in the transverse axis, the posterior wall is described as being the most technically difficult [40]. However, in the cur- 
$>$ Table 3 Results of the current study.

\begin{tabular}{|c|c|c|c|}
\hline & Odds ratio & $P$ & Favors \\
\hline \multicolumn{4}{|l|}{ Curative resection } \\
\hline \multicolumn{4}{|l|}{ Patient prognostic factors } \\
\hline - Female vs male & $1.20(0,93,1.57)$ & $P=0.17$ & Female \\
\hline - Elderly vs non-elderly & $1.00(0.61,1.64)$ & $P=1.00$ & \\
\hline - $\mathrm{BMI} \geq 25$ vs $<25$ & $0.84(0.57 ; 1.26)$ & $P=0.41$ & $\mathrm{BMI} \geq 25$ \\
\hline - HP positive vs HP negative & $0.59(0.36,0.97)$ & $P=0.04$ & HP positive \\
\hline \multicolumn{4}{|l|}{ Lesion prognostic factors } \\
\hline - Non-upper vs upper & $1.41(0.93,2.14)$ & $P=0.10$ & Non-upper \\
\hline - Differentiated vs undifferentiated & $0.10(0.07,0.15)$ & $P<0.00001$ & Differentiated \\
\hline - Non-posterior vs posterior & $1.35(0.81,2.27)$ & $P=0.25$ & Non-posterior \\
\hline - Non-depressed vs depressed & $1.21(0.99,1.49)$ & $P=0.07$ & Non-depressed \\
\hline - Non-ulcer vs ulcer & $3.91(2.31,6.60)$ & $P<0.00001$ & Non-ulcer \\
\hline - Size $<20 \mathrm{~mm}$ vs $>20 \mathrm{~mm}$ & $3.61(2.67,4.88)$ & $P<0.00001$ & $<20 \mathrm{~mm}$ \\
\hline - Mucosa/SM1 vs submucosa, m & $0.02(0.00,0.69)$ & $P=0.03$ & Mucosa/SM1 \\
\hline \multicolumn{4}{|l|}{ Procedure prognostic factors } \\
\hline - Non-bleeding vs bleeding & $2.13(1.56,2.93)$ & $P<0.00001$ & Non-bleeding \\
\hline \multicolumn{4}{|l|}{ Cure } \\
\hline \multicolumn{4}{|l|}{ Patient prognostic factors } \\
\hline - Female vs male & $1.62(1.33,1.97)$ & $P<0.00001$ & Female \\
\hline - Non-elderly vs elderly & $1.49(0.69,3.23)$ & $P=0.31$ & Non-elderly \\
\hline \multicolumn{4}{|l|}{ Lesion prognostic factors } \\
\hline - Differentiated vs undifferentiated & $0.71(0.20,2.51)$ & $P=0.60$ & \\
\hline . SRC vs PDR & $2.24(0.44,11.35)$ & $P=0.33$ & SRC \\
\hline - Depressed vs non-depressed & $1.12(0.72,1.74)$ & $P=0.60$ & \\
\hline - Non-ulcer vs ulcer & $0.91(0.21,3.95)$ & $P=0.90$ & \\
\hline - Upper vs non-upper & $1.25(0.97,1.63)$ & $P=0.90$ & Non-upper \\
\hline - Size $<20$ mm vs > 20 mm & $1.68(0.82,3.45)$ & $P=0.16$ & $<20 \mathrm{~mm}$ \\
\hline - Size $<30$ mm vs > 30 mm & $2.22(0.56,8.81)$ & $P=0.326$ & $<30 \mathrm{~mm}$ \\
\hline - Mucosa/SM1 vs SM2 & $0.08(0.02,0.39)$ & $P=0.002$ & Mucosa/SM1 \\
\hline \multicolumn{4}{|l|}{ Metachronous tumor } \\
\hline - Female vs male & $1.64(1.32,2.03)$ & $P<0.00001$ & Female \\
\hline - HP eradicated vs HP persistent & $1.37(0.95,1.97$ & $P=0.09$ & HP eradicated \\
\hline - HP negative vs HP persistent & $1.61(0.90,2.89)$ & $P=0.11$ & HP negative \\
\hline - HP negative vs eradicated & $0.88(0.21,3.61$ & $P=0.85$ & \\
\hline - Gastric atrophy-mild vs severe & $0.60(0.45,0.81)$ & $P=0.006$ & Mild gastric atrophy \\
\hline - Pepsinogen ratio & $2.29(1.47,3.57)$ & $P=0.0002$ & PGI: PG $\|>3$ \\
\hline
\end{tabular}


rent study, we did not see any difference in success of curative resection. Experienced ESD endoscopists for whom difficult locations are no longer challenging were involved in most of the published studies. This may possibly explain the absence of difference in the success of curative resection.

Regarding macroscopic type, there was no difference in success of curative resection in depressed vs. non-depressed lesions. However, ulcer presence is a strong predictor of nonresectability, according to our study, corroborating the expanded criteria [30]. Ulcer presence may prevent adequate margin delimitation due to the inflammatory process, as well as hindering adequate depth assessment. However, once curative resection is obtained, ulcer presence does not interfere with long-term cure [42].

Lesions smaller than $20 \mathrm{~mm}$ have a higher chance of curative resection than those larger than $20 \mathrm{~mm}$. On the other hand, size was not statistically significant when evaluating long-term cure. Therefore, if a tumor was removed with curative resection criteria, size itself was not relevant for follow-up and should not be used alone to contraindicate endoscopic resection.

Resection is more likely to be curative and over the long term in tumors restricted to the mucosal layer and superficial submucosa, corroborating previous studies [30].

Interestingly, $\boldsymbol{H}$. pylori infection status affected the success of curative resection. One hypothesis to justify this observation could be that inflammation caused by this pathogen around the neoplastic and dysplastic tissue could help in its delimitation, which is different than the hypothesis proposed by Horiuchi et al. [43], who postulated that inflammation could impair resection of some tumors, especially undifferentiated ones. However, only two studies could be used in the analysis after outlier withdrawal, which may limit generalization of this result. It was not possible to analyze the impact of $H$. pylori o long-term cure due to absence of adequate articles to include in the analysis.

Two articles in our study analyzed BMI of patients who underwent ESD for early gastric cancer $[18,44]$. The rationale for this analysis is that obesity is associated with several intraoperative and postoperative complications [44], and it is postulated that it may also influence endoscopic resections. Adipose tissue is often found in gastric submucosa during ESD. Excessive adipose tissue may prevent recognition of vessels in the submucosa, a fact that precludes preventative coagulation and may increase bleeding, thereby making it difficult to perform the procedure [44]. In our study, there was no difference in curative resection between obese and non-obese patients. However, the small number of articles and use of the Asian classification for obesity [17] substantially limit generalization of these data.

Although $\mathrm{H}$. pylori is considered a carcinogen because it induces chronic inflammation and leads to development of preneoplastic lesions, many studies attempt to correlate $H$. pylori infection with a higher incidence of metachronous tumor. There is substantial discussion and divergence in the literature [45-54] on this topic. Whether eradication of $H$. pylori can actually facilitate regression of precancerous lesions, such as atrophy and metaplasia, is unknown $[49,50]$. In a prospective study, eradication of $H$. pylori reduced incidence of gastric cancer only when there were no pre-neoplastic lesions [51]. Within this framework, we sought to identify whether infectious status would imply worse prognosis in relation to incidence of metachronous tumors. There was no difference in incidence of metachronous tumors when comparing patients who were infected, treated, and cured of $H$. pylori. The difference was significant only in relation to degree of atrophy; increased atrophy was associated with greater probability of a metachronous tumor $[52,53]$. In a meta-analysis performed by Xiao [54], it was found that eradication of the pathogen only prevented metachronous tumor occurrence in early stages of carcinogenesis. In other words, chronic inflammation was not enough to cause a severe degree of atrophy. This notion is supported by our evidence that there is a higher chance of metachronous tumor with significant gastric atrophy when compared to mild gastric atrophy.

The gastric mucosa is known to produce two types of pepsinogen (PGI and PGII). In the presence of atrophic gastritis, PGI production by oxyntic cells is lower, while PGIl production remains relatively constant. Reduced serum levels of pepsinogen I $(<70 \mathrm{mg} / \mathrm{L})$ and a PGI/PGIl ratio of less than three are useful in identifying patients with atrophic gastritis. Because significant atrophy is a significant risk factor for incidence of metachronous tumor, the ratio of pepsinogen I and II also directly reflects risk for metachronous tumor. Two studies included in this meta-analysis evaluated incidence of metachronous tumor with these ratios $[55,56]$. The rate between pepsinogen I and II less than three is a risk factor for tumor recurrence, making it crucial to closely follow these patients.

One limitation of this review is that none of the selected studies was a randomized trial, because there are no randomized trials in the literature that evaluate the studied prognostic factors. The selected studies were retrospective cohorts, and therefore, susceptible to selection biases, which may have been mitigated by inclusion of only high-quality work by the NewCastle Score. The included studies vary considerably in relation to number of patients and lesions, as well as high variability in follow-up time, which could explain the high heterogeneity observed in some analyses. However, the heterogeneity limit of $30 \%$ with calculation of sensitivity analysis from this point made it possible to reduce the impact of this variation in our analysis. Most studies (45 from 46) are from Asian countries, which makes it impossible to accurately generalize the results to the Western population. Due to the high incidence of this cancer, some Asian countries, such as Korea and Japan, have an efficient screening program, which provides useful data for future studies. Regarding the histological analysis, Choi and colleagues [18] emphasize that pathological diagnosis may differ significantly between observers, a fact that corroborates the necessity of careful analysis. Western and Korean countries use the World Health Organization classification [57], which differ from some histological subdivisions of Japanese classification [3]. The divergence between western and eastern pathologists was partially resolved with use of the Vienna classification [58], however, there is still heterogeneity among pathologists. Thus, authors should carefully consider different histologies in the efforts for classification of prognostic factors. 
Despite the aforementioned limitations, this meta-analysis is meaningful given the robust correlation of several prognostic factors in a cancer with significant morbidity and mortality. Awareness of prognostic factors of gastric cancer in the early stages will enable clinicians to predict the utility of endoscopic treatment. Also, such prognostic factors allow for patient counseling on the probabilities of an expected outcome based on evidence, according to that individual's unique cancer metrics. We hope this study will lead to new avenues of research and updated guidelines for the scientific community.

\section{Conclusion}

Lesions presenting differentiated histology, without ulceration, smaller than $20 \mathrm{~mm}$, and with invasion over SM1 are associated with a higher rate of curative resection. Absence of bleeding during endoscopic resection and presence of $H$. pylori infection are also factors suggestive of good prognosis related to curative resection. In relation to long-term cure, female sex and invasion of just SM1 increases curative rates. Female gender is a protective factor for developing a metachronous tumor. Severe gastric atrophy and PG I: PGII less than three are risk factors for incidence of metachronous tumor. Awareness of prognostic factors associated to ESD will aid in selection of patients with a higher probability to benefiting from treatment and allow follow-up of these individuals to be customized.

\section{Competing interests}

The authors declare that they have no conflict of interest.

\section{References}

[1] Cutsem EV, Sagaert X, Topal B et al. Gastric cancer. Lancet 2016; 388 : 2654-2664

[2] Kim J, Kim SS. Early Detection is Important to Reduce the Economic Burden of Gastric Cancer. J Gastric Cancer 2018; 18: 82-89

[3] Japanese Gastric Cancer Association. Japanese classification of gastric carcinoma: 3rd English edition. Available from [homepage on the internet]: http://www.jgca.jp/pdf/JGCA_Jpn_Classification_3rd_Eng. pdf

[4] Choi JM, Kim JG, Yang HJ et al. Endoscopic predictors for undifferentiated histology in differentiated gastric neoplasms prior to endoscopic resection. Dig Endosc 2018; 30: 624-632

[5] Choi YK, Ahn JY, Kim DH et al. Efficacy and safety of endoscopic submucosal dissection for gastric neoplasms in patients with compensated liver cirrhosis: a propensity score-matched case-control study. Gastrointest Endosc 2018: 87

[6] Ono H, Yao K, Fujishiro M et al. Guidelines for endoscopic submucosal dissection and endoscopic mucosal resection for early gastric cancer. Digest Endosc 2015; 28: 3-15

[7] Kondo A, De Moura EG, Bernardo WM et al. Endoscopy VS Surgery in the treatment of early gastric cancer: Systematic review. World J Gastroenterol 2015; 21: 13177-13187

[8] Goh PG, Jeong HY, Kim MJ et al. Clinical outcomes of endoscopic submucosal dissection for undifferentiated or submucosal invasive early gastric cancer. Clin Endosc 2011; 44: 116-122
[9] Han JS, Jang JS, Choi SR et al. A study of metachronous cancer after endoscopic resection of early gastric cancer. Scandinavian J Gastroenterol 2011; 46: 1099-1104

[10] Lee JY, Cho KB, Kim ES et al. Risk factors for local recurrence after en bloc endoscopic submucosal dissection for early gastric cancer. World J Gastrointest Endosc 2016; 8: 330-337

[11] Libanio D, Pimentel-Nunes P, Afonso LP et al. Long-term outcomes of gastric endoscopic submucosal dissection: focus on metachronous and non-curative resection management. GE Port J Gastroenterol 2017; $24: 31-39$

[12] Min BH, Kim ER, Kim KM et al. Surveillance strategy based on the incidence and patterns of recurrence after curative endoscopic submucosal dissection for early gastric cancer. Endoscopy 2015; 47: 784 793

[13] Shindo Y, Matsumoto S, Miyatani H. Risk factors for postoperative bleeding after gastric endoscopic submucosal dissection in patients under antithrombotics. World J Gastrointest Endosc 2016; 8: 349356

[14] Nobre MRC, Bernardo WM, Jatene FB. The clinical practice based on evidence. Part I - Constructed clinical issues. Rev Assoc Med Bras 2003; 49: 445-449

[15] The Newcastle-Ottawa Scale (NOS) for assessing the quality of nonrandomised studies in meta-analyses. Available from: http://www. ohri.ca/programs/clinical_epidemiology/oxford.asp

[16] Ono H, Yao K, Fujishiro M et al. Guidelines for endoscopic submucosal dissection and endoscopic mucosal resection for early gastric cancer. Digest Endosc 2015; 28: 3

[17] WHO. The Asia-pacific Perspective: Redefining Obesity and Its treatment. Sydney: Health Communications Australia; 2000

[18] Choi IJ, Lee NR, Kim SG et al. Short-term outcomes of endoscopic submucosal dissection in patients with early gastric cancer: a prospective multicenter cohort study. Gut Liver 2016; 10: 739-748

[19] Ministry of Health, Labour and Welfare. Comparison of the average life span. Avaiable from: http://www.mhlw.go.jp/english/wp/wphw9/dl/01e.pdf

[20] Iwai N, Dohi O, Naito Y et al. Impact of the Charlson comorbidity index and prognostic nutritional index on prognosis in patients with early gastric cancer after endoscopic submucosal dissection. Digest Endosc 2018; 30: 616-623

[21] Katsube T, Murayama M, Yamaguchi K et al. Additional surgery after non-curative resection of esd for early gastric cancer. Anticancer Res 2015; 35: 2969-2974

[22] Kato M, Michida T, Kusakabe A et al. Safety and short-term outcomes of endoscopic submucosal dissection for early gastric cancer in elderly patients. Endosc Int Open 2016; 04: E521-E526

[23] Isomoto H, Ohnita K, Yamaguchi N et al. Clinical outcomes of endoscopic submucosal dissection in elderly patients with early gastric cancer. Europ J Gastroenterol Hepatol 2010; 22: 311-317

[24] Toyokawa T, Fujita I, Morikawa T et al. Clinical outcomes of ESD for early gastric neoplasms in elderly patients. Eur J Clin Invest 2011; 41: 474-478

[25] Yang H], Kim SG, Lim JH et al. Novel risk stratification for metachronous recurrence after curative endoscopic submucosal dissection for early gastric cancer. Gastrointest Endosc 2018; 87: 419-428

[26] Zhang Y, Huang L, Li L. Endoscopic submucosal dissection for early gastric neoplasms in elderly patients. J Laparoendosc Advanced Surgl Tech 2014; 24: 391-398. doi:10.1089/lap.2013.0572

[27] Nam HS, Choi CH, Kim SJ et al. Endoscopic predictive factors associated with local recurrence after gastric endoscopic submucosal dissection. Scandinavian J Gastroenterol 2018; 1484153: doi:10.1080/ 00365521 
[28] Lin ], Zhang Y, Xue M et al. Endoscopic submucosal dissection for early gastric cancer in elderly patients: a meta-analysis. World J Surg Oncology 2015; 13: 293

[29] Sumiyoshi T, Kondo H, Fujii R. Short- and long-term outcomes of endoscopic submucosal dissection for early gastric cancer in elderly patients aged 75 years and older. Gastric Cancer 2017; 20: 489-495

[30] Gotoda T, Yanagisawa A, Sasako M et al. Incidence of lymph node metastasis from early gastric cancer: estimation with a large number of cases at two large centers. Gastric Cancer 2000; 3: 219-225

[31] Goto A, Nishikawa J, Hideura E et al. Lymph node metastasis can be determined by justtum or depth and lymphovascular invasion in early gastric cancer patients after endoscopic submucosal dissection. Europ J Gastroenterol Hepatol 2017; 29: 1346-1350

[32] Nakanishi H, Kurosaki M, Takahashi Y et al. Pretreatment gastric lavage reduces postoperative bleeding after endoscopic submucosal dissection for gastric neoplasms. PLoS ONE 2016; 11: e0149235. doi:10.1371/journal.pone.0149235

[33] Park CH, Kim EH, Kang JH et al. Low incidence of synchronous or metachronous tumors after endoscopic submucosal dissection for early gastric cancer with undifferentiated histology. PLoS ONE 2016; 11: e0147874. doi:10.1371/journal.pone.0147874

[34] Jeon HK, Lee SJ, Kim GH et al. Endoscopic submucosal dissection for undifferentiated-type early gastric cancer: short- and long-term outcomes. Surg Endoscopy 2018; 32: 1963-1970

[35] Zhao X, Cai A, Xi H et al. Predictive factors for lymph node metastasis in early gastric cancer with signet ring cell histology: a meta-analysis. ANZ J Surg 2017; 87: 981-986

[36] Horiuchi Y, Fujisaki J, Yamamoto $\mathrm{N}$ et al. Mixed poorly differentiated adenocarcinoma in undifferentiated? type early gastric cancer predicts endoscopic noncurative resection Gastric Cancer 2018; 21: 689-695

[37] Horiuchi Y, Fujisaki J, Yamamoto $N$ et al. Undifferentiated-type component mixed with differentiated-type early gastric cancer is a significant risk factor for endoscopic non-curative resection. Digest Endosc 2018; 30: 624-632

[38] Han JP, Hong S], Kim HK et al. Long-term outcomes of early gastric cancer diagnosed as mixed adenocarcinoma after endoscopic submucosal dissection. J Gastroenterol Hepatol 2015; 30: 316-320

[39] Bang CS, Ho Baik G. Endoscopic submucosal dissection for early gastric cancer with undifferentiated-type histology: A meta-analysis. World J Gastroenterol 2015; 21: 6032-6043. doi:10.3748/wjg.v21. i19.6032

[40] Yoon JY, Shim CN, Chung SH et al. Impact of tumor location on clinical outcomes of gastric endoscopic submucosal dissection. World J Gastroenterol 2014; 20: 8631-8637

[41] Kim EH, Park JC, Song IJ et al. Prediction model for non-curative resection of endoscopic submucosal dissection in patients with early gastric cancer. Gastrointest Endosc 2017; 85: 976-983

[42] Toyakawa T, Inaba T, Omote S et al. Risk factors for non-curative resection of early gastric neoplasms with endoscopic submucosal dissection: Analysis of 1,123 lesions. Experiment Therap Med 2015; 9: 1209-1214
[43] Horiuchi Y, Fujisaki J, Yamamoto $N$ et al. Diagnostic accuracy demarcation using magnifying endoscopy with narrow? band imaging for Helicobacter pylori uninfected undifferentiated type early gastric cancer Gastric Cancer 2018; 21: 988-997

[44] Kang D, Ha SE, Park JM et al. Body mass index and clinical outcomes from endoscopic submucosal dissection of gastric neoplasia. Dig Dis Sci 2017; 62: 1657-1665

[45] Abe S, Oda I, Suziki $\mathrm{H}$ et al. Long-term surveillance and treatment outcomes of metachronous gastric cancer occurring after curative endoscopic submucosal dissection. 2015; 47: 1113-1118

[46] Jung S, Park CH, Kim EH et al. Preventing metachronous gastric lesions after endoscopic submucosal dissection through Helicobacter pylori eradication. J Gastroenterol Hepatol 2015; 30: 75-81

[47] Machata Y, Nakamura S, Fujisawa K et al. Long-term effect of Helicobacter pylori eradication on the development of metachronous gastric cancer after endoscopic resection of early gastric cancer. Gastrointest Endosc 2012; 75: 39-46

[48] Sugimoto T, Yamaji Y, Sakitani K et al. Neutrophil infiltration and the distribution $\mathrm{f}$ intestinal metaplasia is associated with metachronous gastric cancer following endoscopic submucosal dissection. Can J Gastroenterol Hepatol 2015; 29: 321-325

[49] Han SJ, Kim SG, Lim JH et al. Long-term effects of helicobacter pylori eradication on metachronous gastric cancer development. Gut Liver 2018; 12: 133-141

[50] Chung CS, Who HS, Chung JW et al. Risk factors for metachronous recurrence after endoscopic submucosal dissection of early gastric cancer. J Korean Med Sci 2017; 32: 421-426

[51] Wong BC, Lam SK, Wong WM et al. Helicobacter pylori eradication to prevent gastric cancer in a high-risk region of China: a randomized controlled trial. JAMA 2004; 291: 187-194

[52] Kim SB, Lee SH, Bae SI et al. Association between Helicobacter pylori status and metachronous gastric cancer after endoscopic resection. World J Gastroenterol 2016; 22: 9794-9802

[53] Jung DH, Kim JH, Lee YC et al. Helicobacter pylori eradication reduces the metachronous recurrence of gastric neoplasms by attenuating the precancerous process. J Gastric Cancer 2015; 15: 246-255

[54] Xiao S, Li S, Zhou S et al. Helicobacter pylori status and risks of metachronous recurrence after endoscopic resection of early gastric cancer: a systematic review and meta-analysis. J Gastroenterol 2019; 54: 226-237

[55] Iguchi M, Kato J, Yoshida T et al. Serum pepsinogen levels can quantify the risk of development of metachronous gastric cancer after endoscopic resection. Int J Cancer 2016; 139: 1150-1156

[56] Kwon Y, Jeon S, Nam S et al. Helicobacter pylori infection and serum level of pepsinogen are associated with the risk of metachronous gastric neoplasm after endoscopic resection. Aliment Pharmacol Ther 2017; 46: 758-767

[57] Hamilton SR, Aaltonen LA. Pathology and genetics of tumours of the digestive system. Lyon: IARC press; 2000

[58] Schlemper R], Riddell RH, Kato Y et al. The Vienna classification of gastrointestinal epithelial neoplasia. Gut 2000; 47: 251-255 